\title{
The Application of Chatbot for Customer Service in E-Commerce
}

\author{
Billy Wibowo ${ }^{1}$, \\ ${ }^{1}$ Computer Science Department, School of Computer Science, \\ Bina Nusantara University, \\ Jakarta, Indonesia 11480 \\ billy.wibowo@binus.ac.id; helen.clarissa@binus.ac.id; dsuhartono@binus.edu
}

\begin{abstract}
The use of intelligent machines such as chatbot have been increasing each day. The main idea that we wanted to address in our paper is that with the capabilities that intelligent machines have it will be able to replace humans in doing a certain task and be able to bring better advantages in the long run. The focus of this study is that we are able to manipulate the ability of chatbot to impersonate how humans interact with each other and will enable it to play a role as to handle the customer service in e-commerce websites or applications which will be an alternative to replace the traditional customer service.
\end{abstract}

Keywords: Chatbot; Self-learning; Deep learning; NLP; customer service.

\section{INTRODUCTION}

Rahman et al. (2017) suggested that chatbots -while still in the era of development- will be the next big thing as a tool in the era of conversational services as the use of it has been growing in the mainstream market. This study explores the use of chatbot in the area of customer service for an e-commerce website rooted on our belief that customer service plays an essential part in the growth of a company. As illustrated in figure 1, we can see that the highest number of predictions regarding the use of chatbot is similar to the job desk that a customer service has which is to understand the problem that a customer may face and give the best response that fulfills the customer expectation (Parasuraman et al., 1991).

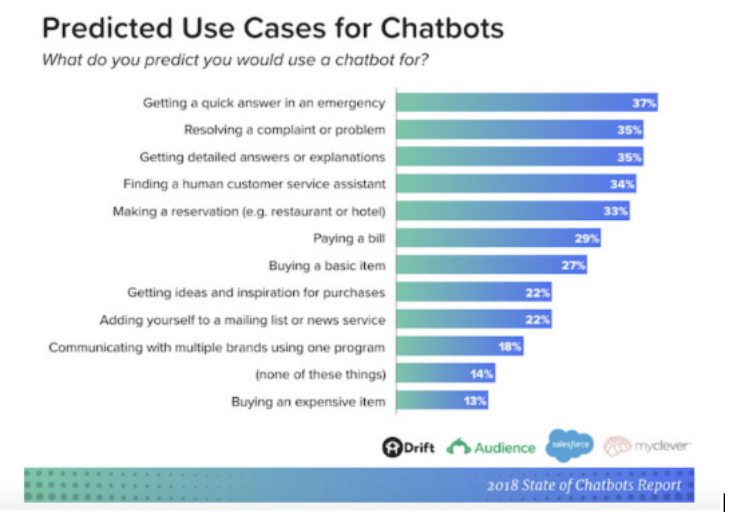

Figure 1. The prediction of chatbot usage per of 2018

The problem that one may encounter while using conventional customer service that is operated by humans such as the time efficiency, the long hold time, conventionality and mistake in information given can easily be solved by the use of chatbot. McGrath (2018) reported that according to IBM, up to $80 \%$ of common customer service questions could easily be answered by a chatbot to multiple customers at once while also reducing company cost up to $30 \%$ with their low maintenance. Another big reason for adopting the use of chatbot is the high interest that people have in conversing with chatbot rather than conventionally especially with millennials where they prefer the least human interaction as possible as they value convenience and generally tend to avoid other humans for service. This is supported by the higher rating satisfaction on most live chat than any of other customer service channels. We also have done a survey to give us more knowledge and confirmation regarding this issue.

The chatbot that we are studying in this paper is a chatbot that will store the information of the product database information such as quantity, color option, size and price and will generate it as an answer when a question 
that contains a keyword is asked. The chatbot that we vision differs from other chatbot in the current market as ours we focus on the ability of the chatbot to adapt to the user's speech pattern and vocabulary because Coperich et al. (2017) suggested, a chatbot that has those abilities believed to be more entertaining and have a better positive view by users in their service performance.

Based on that background the purpose of us writing this paper is to discuss more in depth about the prospect of the idea of customer service chatbot in e-commerce market and how we can improve them to achieve greater results than the previous chatbot in the market in order to carter toward customer satisfaction.

There are many kinds of chatbot concepts and its purpose. For example, the first chatbot to be found in the world in 1966, according to Weizenbaum (1966) ELIZA uses the recognition of phrases or words in the input and then works through pattern matching to search for relevant responses. Then as the time goes by, the industry of chatbot develops by using help of Natural Language Processing and Deep Learning. Unlike ELIZA, a chatbot named ALICE developed in 2009 uses Artificial Intelligence Markup Language(AIML) and is better than ELIZA from speed and interoperability (Jwala et al., 2019).

But then the purpose of chatbot is not only for responding the user input anymore, but also for responding an e-commerce website. According to Siddharth (2015) chatbot can be using proposed system which the user can interact with the bot using Natural Language and the chatbot can suggest any product or any particular brand when the user input a budget that the user have right now. More example of chatbot for e-commerce website is SuperAgent (Lei, 2017). The differences between SuperAgent and chatbot that Siddarth wrote is the SuperAgent can separate the type of chat, like chit-chat type of chat, fact QA, text QA, and FAQ search. SuperAgent can respond well to many of them by using crawlers to crawl the html information when the product page is first visited then split them into many types of chat. SuperAgent also uses NLP and machine learning techniques. For customer service, there is chatbot named Lingke (Zhu et al., 2018) that uses coreference resolution and document separation(using Stanford CoreNLP), target sentences retrieval(using Apache Lucene), candidate responses generation, and dialogue manager. The dialogue manager is divided by three aspects, that is self-matching attention, response selection, and chit-chat response generation. The other example of chatbot for customer service is a chatbot for customer service on social media $(\mathrm{Xu}$, et al., 2017). This paper discusses customer service chatbot via deep learning techniques that can be implemented to sequence-to-sequence learning(to recognize order of input sentence) and word embedding(to represent few words in a continuous space and to differentiate between words). To create the system, there are many steps to perform. First, clean the data then tokenize the data, later on generate word-embedding features and last but not least, train LSTM (Long Short-term Memory) network.

\section{METHODS}

\subsection{The Chatbot Concept}

Our chatbot concept is to have a bot that is easily accessible in the ecommerce website or mobile application of the e-commerce which functions to guide and help users in knowing the detailed information of the product, and handle simple problems that users might encounter while using the application. As for now our chatbot concept only will be based on typed conversation between customer and the chatbot.

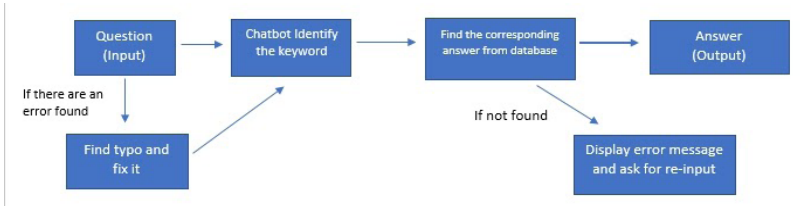

Figure 2. The chatbot flowchart from Input to Output

When a Customer opens the chat box of the chatbot they are asked to enter an input a question which the bot will later process by first identifying the keyword from the sentences. How chatbot usually operate in finding keyword is that they separate each word in the sentences that the user have inputted and giving them their syntactic category such as verb and noun and from that the bot will remove the unnecessary part of the sentences and only operated based on words that have been included as a trigger or a keywords. from there the bot can know what the customer want and will ask and know what next to process, if the bot unable to find the necessary keyword to do their task they will ask for the user to re input. If the bot don't have the completed information then the bot will then ask a question to the user in order to know more about the more specific request that the user intended to ask. When the needed information is collected by the bot the bot will search the answer from their database and give the output as an answer to the user. To improve the correctness of answers given by the chatbot, the concept model of NLP and deep learning method is applied. This will allow the bot to be able understand natural language, identify meaning and emotion to give the most suitable answers that the user searching with much larger accuracy (as shown in Figure 2).

The issues that usually occur when building a datadriven chatbot are to overcome the challenge to model a natural conversation and in order to also achieve a clean, systematic and unbiased dataset may become an obstacle to the chatbot improvement in processing the natural language.

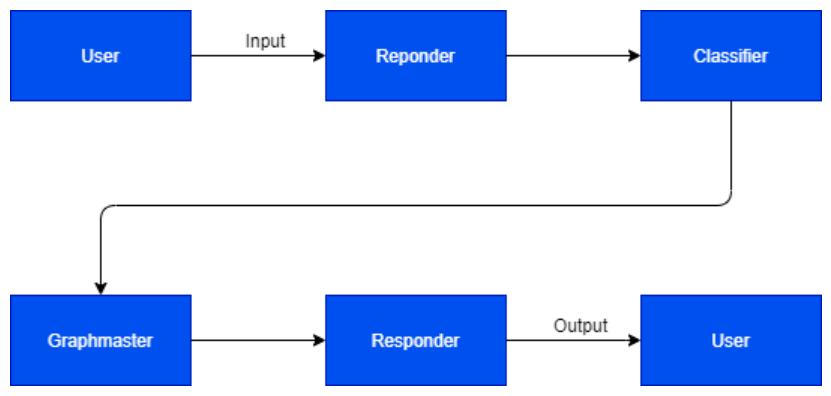

Figure 3. Components of a chatbot 
Commonly a chatbot can be divided into three parts (as shown in Figure 3):

1. Responder which is the bridge or connector between bot and user that transfers data to be processed by the bot.

2. Classifier which later processes and filters to be segmented into smaller components.

3. Graphmaster which does the pattern matching.

\section{RESULTS AND DISCUSSION}

For this part we use a survey questionnaire to find out about people's experience about chatbot and to find out about their interest in chatbot concept.
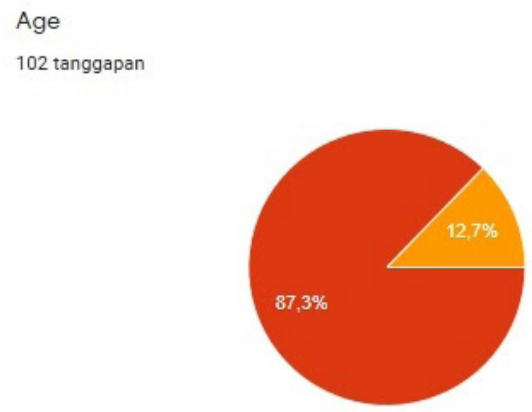

Figure 4. Survey about Age

From figure 4 on the above we know that there are a total of 102 random respondents with $87.3 \%$ of them being a teenage range from age $17-20$ and the other $12.7 \%$ being more than 20 years old.

Have you ever used a chat bot before

102 tanggapan

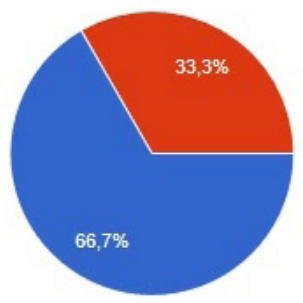

Yes No

Figure 5. Survey about Experience

From figure 5 above asking about their experience, the data show that from all the respondents most of them have used a chatbot before while the smaller percentage $33.3 \%$ never actually used a chatbot as far as they are concerned. are you interested in a concept of chat bot that can help you answer details of products in a ecommerce platform ?

102 tanggapan

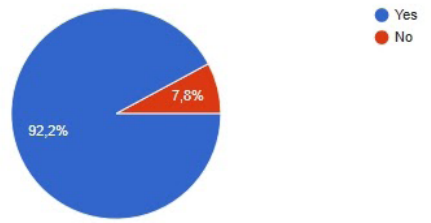

Figure 6. Survey about Interest

And last but not least from figure 6 on the above when being asked if they are interested in the application of chatbot in an e-commerce platform as a customer service most of them give a positive response with them believing that chatbot will be able to outperform the customer service in that industry right now.

From the survey that we conducted on April $1^{\text {st }} 2020$, there are counted 102 respondent from the people with majority being students that is doing their study in Bina Nusantara University that fills out the form with the result that can be summarized that while the use of chatbot is still consider to be uncommon for many of the respondent but majority of people between the age group or 17 and older is not against in the idea of using chatbot as a customer service this conclude that there will be high interest rate from the user if we were to apply the chatbot in market today and it may bring higher engagement rate between user and the website opening up more chances of user to buy from that website. This proves that many youth are more leaning in supporting the use of technology in their daily life as they believe it is more efficient and convenient to use it in their daily life and in this case is to assist them in finding information.

With the consideration of those that may find chatbot to be a new thing we have designed an interface that is simple and easy to understand even by a new user that is unfamiliar with chatbot before as it uses the same interface as a messenger . below are the example of chatbot chat interface that is happening between the bot and a user : 


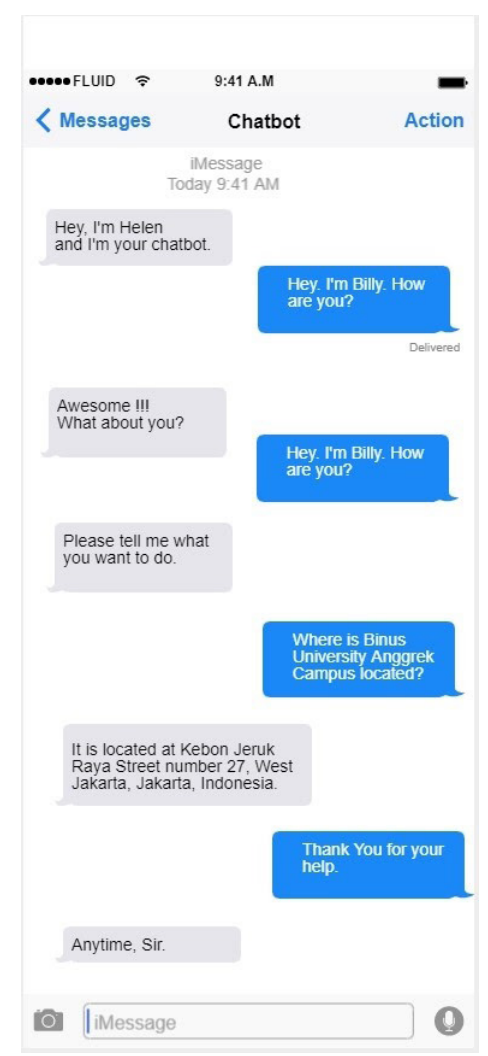

Figure 7. Example of Chatbot Design

\section{CONCLUSION}

From this paper we know that chatbot is very useful for human life and people nowadays are becoming more and more interested in it. In this paper, we give the idea and concept to develop a chatbot that is able to access e-commerce websites or mobile applications easily and able to work as what a customer service would do. That is to answer questions, provide details and information and help in problems that users may face. Compared to conventional customer service chatbots take advantage of large-scale, publicly available, and crowd-sourced customer data. It also works in a more time and cost-efficient way but still able to satisfy customer demand.

For future prospects itself chatbot is believed to be more commonly used in daily life especially with advanced technology in this millennial era. Which means it will give the company or anyone that uses this chatbot as customer service a long-term advantage, profit incomes, and many other benefits for e-commerce.

\section{REFERENCES}

Aggarwal , V., Jain, A., Khatter, H., \& Gupta, K. (2019). Evolution of Chatbots for Smart Assistance. International Journal of Innovative Technology and Exploring Engineering, pp. 77-83.

Asadi, A.-r., \& Hemadi, R. (2018). Design and Implementation of a chatbot for e-commerce. Information Communication Technology and Doing Busi- ness, pp. 1-10.

Coperich, K., Cudney, E., \& Nembhard, H. (2017). Continuous improvement study of chatbot technologies using a human factors methodology. In Proceedings of the 2017 Industrial and Systems Engineering Conference.

Dahiya, M. (2017). A Tool of Conversation: Chatbot. International Journal of Computer Sciences and Engineering (JCSE). pp. 1-4

Elsholz, E., Chamberlain, J., \& Kruschwitz, U. (2019). Exploring Language Style in Chatbots to Increase Perceived Product Value and User Engagement. Conference on Human Information Interaction and Retrieval, pp. 301-305.

Florian Johannsen, S. L. (2018). Comparison of Commercial Chatbot Solution for Supporting Customer Interaction. Association for Information Systems,pp. 937-947.

Haan, H. d. (2018). Chatbot Personality and Customer Satisfaction. Utrecht University, pp. 1-40.

Jwala, K., Sirisha, G., \& Padma Raju, G. (2019). Developing a Chatbot using Machine Learning. International Journal of Recent Technology and Engineering, pp. 89-92.

Khan , R. (2017). Standardized Architecture for Conversational Agents a.k.a. ChatBots. International Journal of Computer Trends and Technology, pp. 114-121.

Lei Cui, S. H. (2017). SuperAgent: A Customer Service Chatbot for E-commerce Websites. Association for Computational Linguistics, pp. 97-102.

McGrath, R. (Author). (2018, 3 May). How To Improve Customer Service With Chatbot. Retrieved June 15th 2020 from https://chatbotsmagazine.com/ ill-never-buy-from-them-again-using-chatbotsto-avoid-bad-customer-service-e6a967360244

Nuruzzaman, Mohammad \& Hussain, Omar. (2018). A Survey on Chatbot Implementation in Customer Service Industry through Deep Neural Networks. 10.1109/ICEBE.2018.00019. pp. 54-61.

Parasuraman, A., Berry, L. L., \& Zeithaml, V. A. (1991). Understanding customer expectations of service. Sloan management review, 32(3), 39-48.

Rahman, A. M., Al Mamun, A., \& Islam, A. (2017). Programming challenges of chatbot: Current and future prospective. In 2017 IEEE Region $10 \mathrm{Hu}-$ manitarian Technology Conference (R10-HTC) (pp. 75-78).

Sameera A. Abdul-Kader, D. J. (2015). Survey on Chatbot Design Techniques in Speech Conversation Systems. (IJACSA) International Journal of Advanced Computer Science and Applications, pp. $72-80$.

Schmidt, R. (2018). Learning System Customer Service 
Chatbot. Georgia Institute of Technology, pp. $1-5$.

Siddharth Gupta, D. B. (2015). An E-Commerce Website based Chatbot. International Journal of Computer Science and Information Technologies (IJCSIT), 6, pp. 1483-1485.

Weizenbaum, J. (1966). ELIZA: A Computer Program for The Study of Natural Language Communication between Man and Machine. Commun.ACM, p. $36-45$.

Xu, A., Liu, Z., Guo, Y., Sinha, V., \& Akkiraju, R. (2017). A New Chatbot for Customer Service on Social Media. Proceedings of the 2017 CHI Conference on Human Factors in Computing Systems at New York, USA, pp. 3506-3510.

Xueming Luo, S. T. (2019). Machines vs. Humans: The Impact of Artificial. Institute for Operations Research and the Management Sciences (INFORMS), pp. 1-12.

Zhu, P., Zhang, Z., Li, J., Huang, Y., \& Zhao, H. (2018). Lingke: A Fine-grained Multi-turn Chatbot for Customer Service. Proceedings of the 27th International Conference on Computational Linguistics: System Demonstrations, At Santa Fe, New Mexico, pp. 108-112.

Zumstein, D., \& Hundertmark, S. (2017). Chatbots - An Interactive Technology for Personalized Communication, Transaction and Services. IADIS International Journal, pp. 96-109. 